\title{
Velocity Map Imaging for Photocathode Characterization
}

\author{
Hong Ye ${ }^{1,2, *}$, Sebastian H. Trippel ${ }^{1,3}$, Michele Di Fraia ${ }^{1}$, Arya Fallahi ${ }^{1}$, \\ Oliver D. Mücke ${ }^{1,3}$, Jochen Küpper ${ }^{1,2,3}$, and Franz X. Kärtner ${ }^{1,2,3}$ \\ ${ }^{1}$ Center for Free-Electron Laser Science, DESY, Notkestrasse 85, 22607 Hamburg, Germany \\ ${ }^{2}$ Department of Physics, University of Hamburg, Luruper Chaussee 149, 22761 Hamburg, Germany \\ ${ }^{3}$ The Hamburg Center for Ultrafast Imaging, University of Hamburg, Luruper Chaussee 149, \\ 22761 Hamburg, Germany \\ *hong.ye@desy.de
}

\begin{abstract}
A velocity map imaging spectrometer (VMI) to characterize the transverse phase space of electron emission from solid surfaces is described. A first instrument test is presented using multi-photon emission from a planar Au surface.
\end{abstract}

OCIS codes: (020.4180) Multiphoton processes; (100.3010) Image reconstruction techniques; (300.6190) Spectrometers

\section{Introduction}

High-brightness ultrafast electron sources are key devices for electron diffractive imaging and free-electron lasers determing respectively the image resolution and the best X-ray beam quality with a given electron beam energy [1]. These techniques are crucially limited by the overall electron beam brightness which depends on the total bunch charge and projected emittance. Tremendous experimental efforts have been and still are devoted to characterize and improve the emittance of the electron beam expected from a photocathode. Lately, strong-field emission from nanostructured photocathodes has attracted much attention, because of its potential to enable nanoscale time-resolved diffractive electron microscopy as well as temporally and spatially coherent X-rays [2]. Thus characterization of emittance, in many instances the transverse phase space of the emitted electrons, is of central importance to support claims made. In this paper, we discuss the construction of a velocity map imaging (VMI) spectrometer that enables transverse phase space characterization of electrons released from cathodes. To confirm its functionality, we measure the energy distribution of electrons photoemitted from an Au planar surface irradiated by intense 45 fs laser pulses centered at $800 \mathrm{~nm}$.

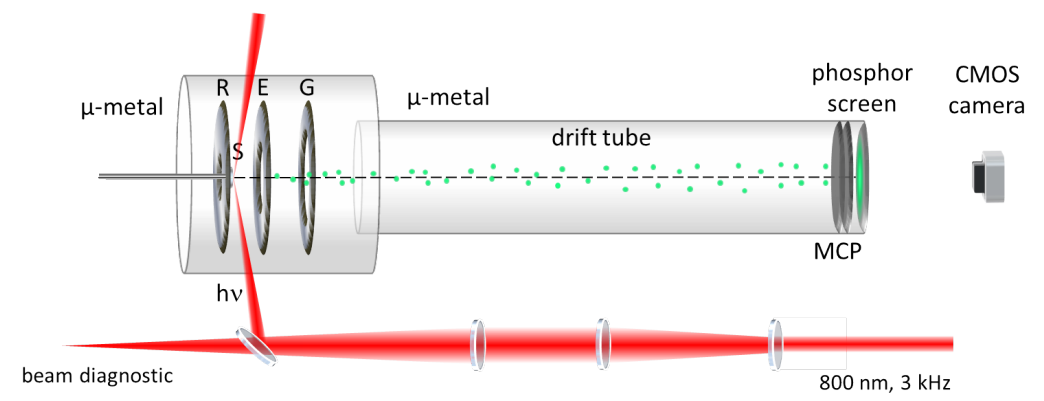

Fig. 1. Schematic of the velocity-map-imaging (VMI) setup. R: repeller, E: extractor, G: ground electrode. The red line represents the laser beam. The green dots depict the emitted electrons.

\section{Experimental Setup and Results}

In Fig.1, the VMI spectrometer consists of three electrodes (repeller, extractor, and ground), a 49-cm-long drift tube, followed by the position-sensitive detector assembly made up of a multi-channel plate (MCP), a phosphor screen and a CMOS camera. The electrodes and the drift tube were shielded by $\mu$-metal shield inside vacuum $\left(2 \times 10^{-8}\right.$ mbar $)$. The sample was located in the same plane as the front side of the repeller plate. The focused output of an amplified 3-kHz 800-nm Ti:sapphire laser system was used to illuminate the sample with an incident angle of $83^{\circ}$. The laser 
spot size in the focus was $\sim 35 \mu \mathrm{m} \times 250 \mu \mathrm{m}$ full-width-half-maximum (FWHM) on the sample. The pulse duration was $45 \mathrm{fs}$ measured by frequency-resolved optical gating (FROG). The average laser power used in the experiments was between 6-25 $\mu \mathrm{W}$, which corresponds to a peak intensity of $150-620 \mathrm{MW} / \mathrm{cm}^{2}$. The VMI spectrometer focused the electrons with the same initial transverse velocity vector to the same position on the detector surface. In our experiments, single-shot electron images were read out at a repetition rate of $1 \mathrm{kHz}$, limited by the camera frame rate. Each measurement was averaged for $200 \mathrm{~s}$. Detailed analysis and measurements confirm a $\sim 30 \mu \mathrm{m}$ spatial resolution in the spatial imaging mode and $\sim 2.5 \times 10^{-2} \pi \cdot \operatorname{mrad}$ transverse momentum resolution in the velocity imaging mode.
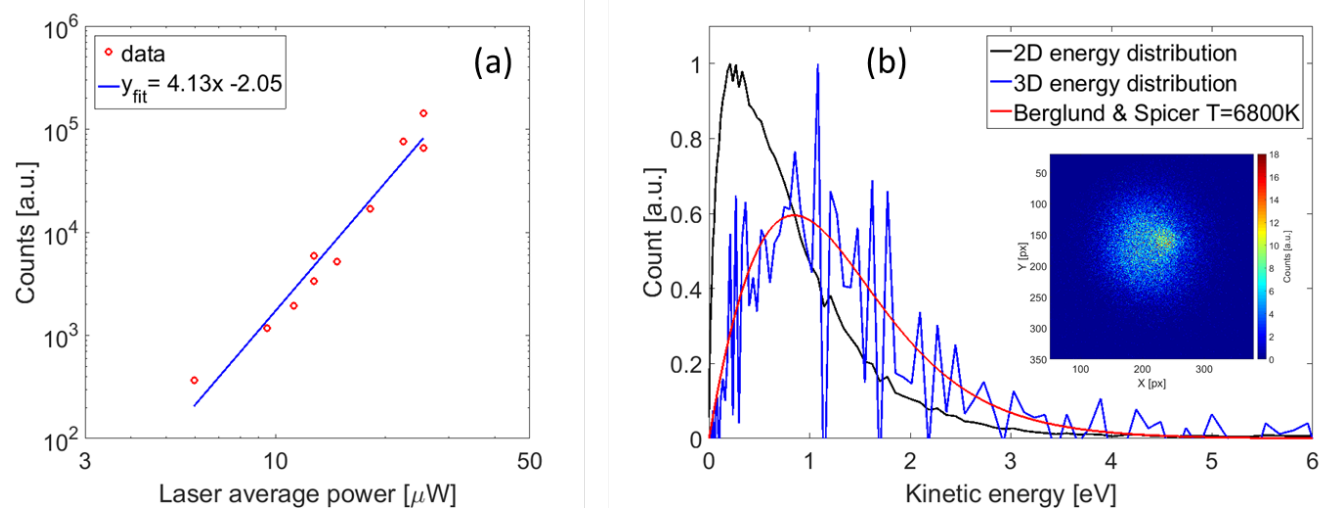

Fig. 2. (a) Photoemitted electron current as a function of laser average power; (b) $2 \mathrm{D}$ kinetic energy distribution (black) extracted from the measured velocity-map image shown as inset. The reconstructed 3D energy distribution (blue) is fitted with the Berglund and Spicer model at $6800 \mathrm{~K}$.

Fig.2(a) shows, on a logarithmic scale, the integrated photoemitted electron yield as a function of average incident laser power. A power-law dependence is observed. According to the Fowler-Dubrige model [3], the data shows a +4 slope in agreement with a 4-th order multiphoton process, indicating that simultaneous absorption of 4 photons with energy of $h v=1.55 \mathrm{eV}$ is required for electrons to overcome the Au work function of $5.31 \mathrm{eV}$. In Fig.2(b), a velocity-map image from an Au planar surface is shown as inset. The data was recorded with an average laser power of $22.5 \mu \mathrm{W}$. The corresponding radial energy distribution of the projected electrons is plotted as a black line. Assuming a theoretically derived cosine dependence of the angle distribution of emitted electrons, the projection of each fixed energy value is uniformly distributed on the detector screen. Therefore, the three dimensional (3D) energy distribution is mathematically reconstructed and shown as a blue line. The Berglund and Spicer model [4] is employed to fit our reconstruction curve. Since the model was derived for single-photon emission, we have implicitly made the assumption that the electron will absorb a sufficient number of photons simultaneously to overcome the barrier and escape to vacuum rather than absorb photons sequentially. An electron temperature of $6800 \mathrm{~K}$ is assumed in the model, which indicates that very hot electrons are generated localized by the femtosecond laser pulse in consistent with the high excess energy deposited into the electronic system. A comparable temperature $\sim 7000 \mathrm{~K}$ was observed by Aeschlimann et al. from $\mathrm{Cu}$ under surface-enhanced multiphoton emission [5]. Since VMI intuitively measures the transverse momentum distribution, assuming there is no correlation between the location of emission and the transverse momentum, the normalized transverse emittance $\varepsilon_{n_{\zeta}}=\frac{\sqrt{\left\langle\zeta^{2}\right\rangle\left\langle p_{\zeta}^{2}\right\rangle}}{m c}$ with $\zeta \in\{x, y\}$ is calculated to be 0.477 and $0.055 \pi \cdot \mathrm{mm} \cdot \mathrm{mrad}$ in $x$ - and $y$ - dimensions, respectively. Our ongoing work aims to characterize the electron emission from nanostructured emitter arrays. The preliminary results observed are promising to characterize electron beam sources with very high brightness.

\section{References}

1. S. D. Mitri, Photonics 2, 317-341 (2015).

2. F. X. Kärtner et al., Nucl. Instr. Meth. Phys. Res. A829, 24-29 (2016).

3. L. A. Dubridge, Phys. Rev. 43, 727 (1933).

4. C. N. Berglund and W. E. Spicer, Phys. Rev. 136, A1030 (1964).

5. M. Aeschlimann et al., J. Chem. Phys. 102, 8606 (1995). 